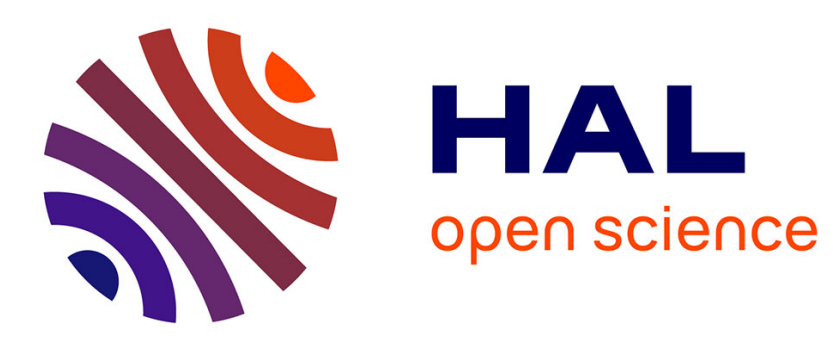

\title{
The dynamic response of a centrifugal pendulum vibration absorber with motion-limiting stops
}

\author{
M. Sharif-Bakhtiar, Steve W Shaw
}

\section{To cite this version:}

M. Sharif-Bakhtiar, Steve W Shaw. The dynamic response of a centrifugal pendulum vibration absorber with motion-limiting stops. Journal of Sound and Vibration, 1988, 126 (2), pp.221-235. 10.1016/0022-460X(88)90237-4 . hal-01635201

\section{HAL Id: hal-01635201 \\ https://hal.science/hal-01635201}

Submitted on 6 Mar 2018

HAL is a multi-disciplinary open access archive for the deposit and dissemination of scientific research documents, whether they are published or not. The documents may come from teaching and research institutions in France or abroad, or from public or private research centers.
L'archive ouverte pluridisciplinaire HAL, est destinée au dépôt et à la diffusion de documents scientifiques de niveau recherche, publiés ou non, émanant des établissements d'enseignement et de recherche français ou étrangers, des laboratoires publics ou privés. 


\title{
THE DYNAMIC RESPONSE OF A CENTRIFUGAL PENDULUM VIBRATION ABSORBER WITH MOTION-LIMITING STOPS†
}

\author{
M. Sharif-Bakhtiar and S. W. Shaw \\ Department of Mechanical Engineering, Michigan State University, \\ East Lansing, Michigan 48824, U.S.A.
}

\begin{abstract}
The effects of motion-limiting stops on the dynamic behavior of a centrifugal pendulum vibration absorber are studied. The equations of motion for the two degrees-of-freedom system are decoupled and an isolated, non-dimensionalized set of equations of motion is obtained for the pendulum which includes the effects of the stops. The results of the analysis provide the existence and stability of the non-linear impacting periodic motions of the pendulum, based on various system parameters. Several properties which are inherent to the system are discovered in this analysis which have not been previously considered. One such property is the coexistence of impacting and non-impacting periodic motions at the desired operating frequency for which the pendulum is designed. The results indicate, however, that such coexistence occurs when the pendulum damping is larger than is reasonable for practical applications. The effect of detuning of the absorber is also considered.
\end{abstract}

\section{INTRODUCTION}

The centrifugal perndulum vibration absorber (CPVA for short) was devised and patented in France about 1935 and at the same time was independently conceived and put into practice by E. S. Taylor [1]. Its purpose was to overcome serious torsional vibrations inherent in geared radial aircraft-engine-propeller systems. However, the device is generally useful for reducing torsional oscillations in rotating shafts in engines and machinery. At a later time, the absorber was modified and incorporated into automotive internal combustion engines in order to alleviate the torsional vibrations of the crank shaft. This was done by integrating the absorber with the crank shaft counterbalance masses [1]. Den Hartog [2] has provided an early account of the CPVA including a discussion of large amplitude, non-linear motions.

The centrifugal pendulum vibration absorber essentially is a tuned absorber whose natural frequency varies in direct proportion to the rotational speed of a rotating shaft, $\Omega$. In the linear range, i.e., small angular displacements of the pendulum absorber, the pendulum can be tuned to reduce the effects of disturbances which are at a frequency proportional to $\Omega$. Figure 1 shows the basic mechanism of the CPVA consisting of a disc, which represents the rotating inertia of the shaft, and the attached pendulum. The pertinent dimensions of the model used for numerical results in this report are listed in the Appendix.

For the usual linear analysis the following assumptions are made: no constraints; the damping is negligible; the excitation is of the form $T(t)=T_{0} \sin \omega t$; the motion of the carrier is a steady state rotation plus a small sinusoidal oscillation, i.e., $\psi=\Omega t+\psi_{0} \sin \omega t$; the pendulum has a steady state solution of the form $\phi=\phi_{0} \sin \omega t$, with $\phi_{0}$ small. It then

† This work was supported in part by NSF Grants MEA-8421248 and MSM-8613294 and by DARPA. 


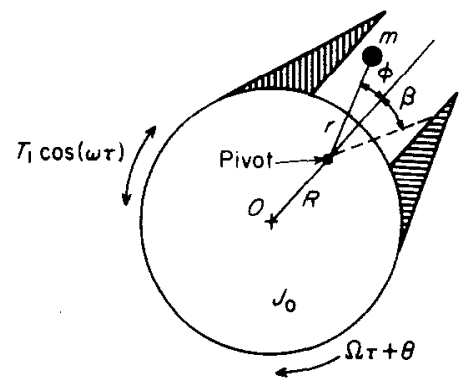

Figure 1. Schematic view of the CPVA.

can be shown, see reference [3] for example, that

$$
\frac{\psi_{0}}{\phi_{0}}=\left(\Omega^{2} \frac{R}{2}-\omega^{2}\right) /\left(\frac{R+r}{r}\right) \omega^{2}
$$

This indicates that, if the frequency of the torsional disturbance on the disc (or carrier) is $j \Omega, j=1,2,3,4, \ldots$, then the pendulum can be tuned such that at the disturbing frequency the rotating disc has no oscillatory motion while the pendulum has a finite oscillatory amplitude. Such disturbances are typical for rotating machines and arise from unbalances or combusion forces, for example. For small angular displacements of the pendulum the disc amplitude is reduced to zero if $\omega=\Omega \sqrt{R / r}$, i.e., $j=\sqrt{R / r}$. In this case the natural frequency of the pendulum corresponding to a constant rate $\Omega, \omega_{p}$, is coincident with the disturbing frequency, $\omega$. This frequency, for which the pendulum is designed, is referred to as the anti-resonance frequency, $\omega_{A R}$. Figure 2 shows a plot of the frequency response of the pendulum and the carrier.

One of the major difficulties in the design and application of the CPVA is excessively large amplitudes of oscillation of the absorber during operation [4]. In other words, absorbers that are thought to be "properly designed" can exhibit dynamics that are inconsistent with the original analysis, especially during transient behavior. An example has been cited by Newland [5] where an engine was designed with pendulums calculated to swing through about 45 degrees. During tests it was found that the vibration absorbers were not functioning properly and, on dismantling the engine, they were found to have been oscillating through a much larger angle than expected, damaging the stops which were set at 75 degrees amplitude.

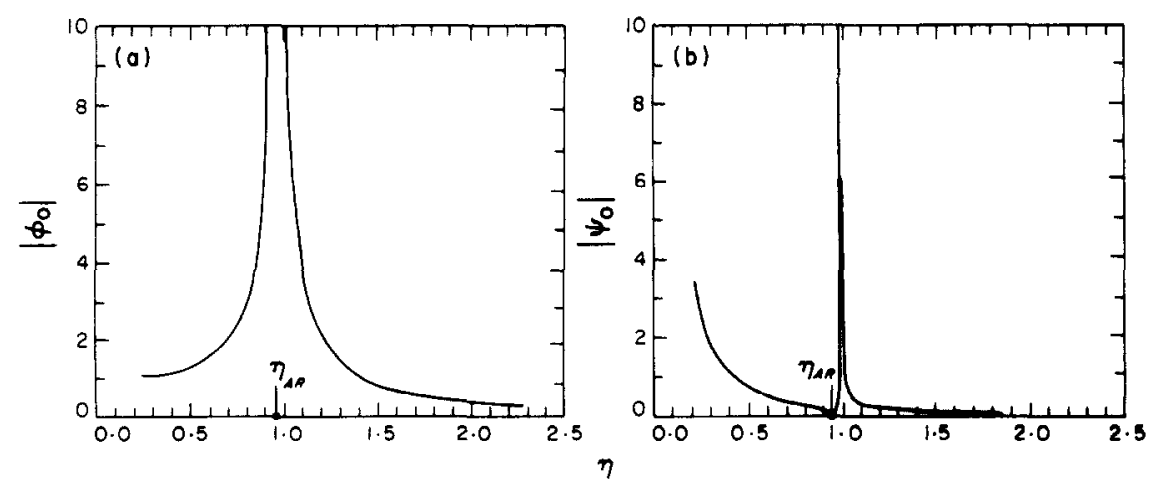

Figure 2. Frequency response of the (a) pendulum without constraints and (b) carrier without constraints. 
Several different approaches have been taken to overcome this problem. One such case is by confining the system such that the pendulum mass, typically in a bifilar design, would move on a non-circular path [6]. Another approach is to use constraints to limit the amplitude of oscillation of the pendulum. The objective of this design is to have the absorber oscillate freely between the two constraints without coming into contact with the stops, except possibly during transient motions.

In order to achieve this objective, the response of the absorber in relation to the constraints and the range of parameters for which impacting and/or non-impacting periodic motions exist is to be studied. In particular, the possibility of coexistence of linear, non-impacting and non-linear, impacting motions at the anti-resonance frequency is of interest. Such coexistence can, in fact, occur and cannot be predicted without a detailed consideration of impacting motions. An absorber thought to be properly designed (i.e., by using only linear analysis and the addition of stops at amplitudes larger than the steady state amplitudes predicted by the linear theory) may encounter impacting steady state dynamics.

It is shown in the following that such coexistence occurs only for damping characteristics of the absorber too large for practical application of the absorber. This result cannot be obtained without a detailed investigation to determine the range of system parameters for which such multi-steady-state dynamic behavior can occur. Also, should the system be subjected to a high frequency disturbance, the response of the absorber with constraints can be quite complicated. This report aims at achieving a better understanding of these issues and at determining the range of system parameters for which a linear absorber with stops can function satisfactorily.

\section{REDUCTION OF THE EQUATIONS OF MOTION}

With the effects of gravity ignored, the large amplitude equations of motion for the system shown in Figure 1 can be written as

$$
\begin{gathered}
{\left[J+m R^{2}+m r^{2}+2 m R r \cos \phi\right] \psi^{\prime \prime}-m r(R \cos \phi+r) \phi^{\prime \prime}} \\
+m R r \phi^{\prime 2} \sin \phi-2 m R r \psi^{\prime} \phi^{\prime} \sin \phi+c_{c} \psi^{\prime}=T(\tau), \\
-m r(R \cos \phi+r) \psi^{\prime \prime}+m r^{2} \phi^{\prime \prime}+c_{p} \phi^{\prime}+m R r \psi^{\prime 2} \sin \phi=0,
\end{gathered}
$$

where $\tau$ denotes time, ( $)^{\prime}$ indicates differentiation with respect to time, $J$ is the polar moment of inertia of the carrier, $c_{p}$ is the pendulum joint damping constant, $c_{c}$ is the carrier bearing dissipation and $T(\tau)$ is the applied torque.

These equations are valid for the case where the pendulum is not in contact with the constraints, i.e., $|\phi|<\beta$. At impact, $|\phi|=\beta$, it is assumed that the contact time is small enough that the law of the conservation of the total angular momentum of the system can be instantaneously applied, i.e.

$$
\begin{gathered}
k_{1} \psi^{\prime+}+k_{2} \phi^{\prime+}=k_{1} \psi^{\prime-}+k_{2} \phi^{\prime-}, \\
k_{1}=-\left[J+m\left(R^{2}+r^{2}+2 m R r \cos \beta\right)\right], \quad k_{2}=m r(R \cos \beta+r),
\end{gathered}
$$

where the (-) and (+) superscripts refer to the times just prior to and after impact, respectively. Another relation describing the dynamics of the impact is determined by the usual definition of the coefficient of restitution, $e$. This relation can be written as

$$
e=\left(V_{p_{n}}^{+}-V_{c_{n}}^{+} /\left(V_{c_{n}}^{-}-V_{p_{n}}^{-}\right)\right.
$$


where $V$ denotes absolute velocity and subscripts $p, c$, and $n$ refer to the pendulum, the carrier, and the direction normal to the line of impact, respectively. Equation (4) can then be expressed, in terms of the angular velocities of the carrier and the pendulum as

$$
\begin{gathered}
e\left[\left(k_{3}+\rho\right) \psi^{\prime+}+k_{4} \phi^{\prime+}\right] /\left[-\left(k_{3}+\rho\right) \psi^{\prime-}-k_{4} \phi^{\prime}\right], \\
k_{3}=-R \cos \alpha-r \cos (\beta-\alpha), \quad k_{4}=r \cos (\beta-\alpha), \\
\alpha=\tan ^{-1}[r \sin \beta /(R+r \cos \beta)], \quad \rho=\left[(R+r \cos \phi)^{2}+r^{2} \sin ^{2} \phi\right]^{1 / 2} .
\end{gathered}
$$

Equations (3) and (5) can be solved simultaneously to obtain equations for $\psi^{\prime+}$ and $\phi^{\prime+}$ in terms of $\psi^{\prime-}$ and $\phi^{\prime-}$ and the system parameters. The resulting relation for $\phi^{\prime+}$ is complicated and it obscures the true dynamics of the impact. An alternative, and more elightening, approach involves use of the geometrical properties of the system. From Figure 1 it can be seen that

$$
k_{3}+\rho=-[R \cos \alpha+r \cos (\alpha-\beta)]+\rho=-(R \cos \alpha+r \cos \alpha \cos \beta+r \sin \alpha \sin \beta)+\rho,
$$

where $\alpha$ is the angle between the line $\phi=0$ and the line from 0 to $m$ at $\phi=\beta$ and $\rho$ is the distance from 0 to $m$ at $\phi=\beta$. Considering

$$
\cos \alpha=(R+r \cos \beta) / \rho, \quad \sin \alpha=(r \sin \beta) / \rho, \rho=\left(R^{2}+r^{2}+2 R r \cos \beta\right)^{1 / 2},
$$

one obtains $k_{3}+\rho=0$, so that equation (5) becomes $e=-\phi^{\prime+} / \phi^{\prime-}$, or

$$
\phi^{\prime+}=-e \phi^{\prime-} \text {, }
$$

which is a familar equation of impact for a single-mass system. This simple result is a natural consequence of the fact that $\phi$ is a relative co-ordinate.

The following assumptions are then made to simplify the free flight equations of motion (1) and (2): (a) the carrier runs at nearly constant speed $\Omega$ with a small time dependent variation, i.e., $\psi=\Omega \tau+\theta$; (b) the carrier damping is assumed negligible, $c_{c}=0$; (c) $\phi$ is small enough that $\sin \phi \cong \phi, \cos \phi \cong 1$; (d) second and higher order terms of $\theta, \phi$ and their derivatives can be neglected (i.e., linearize the equations); (e) the applied torque is sinusoidal in time. With incorporation of these assumptions equations (1) and (2) become

$$
\begin{gathered}
{\left[J+m(R+r)^{2}\right] \theta^{\prime \prime}-m r(R+r) \phi^{\prime \prime}=T(\tau)=T_{1} \sin \omega \tau,} \\
-m r(R+r) \theta^{\prime \prime}+m r^{2} \phi^{\prime \prime}+c_{p} \phi^{\prime}+m R r \Omega^{2} \phi=0 .
\end{gathered}
$$

Eliminating the $\theta^{\prime \prime}$ variable between equations (7) and (8) one obtains

$$
J m r^{2} \phi^{\prime \prime}+c_{p}\left[J+m(R+r)^{2}\right] \phi^{\prime}+m R r \Omega^{2}\left[J+m(R+r)^{2}\right] \phi=m r(R+r) T_{1} \sin \omega \tau
$$

and rescaling the resulting equation yields

$$
\ddot{x}+2 \lambda \dot{x}+x=k \cos \eta t,
$$

where $\left.x=\phi / \beta, t=\left\{R \Omega^{2} / J r\right)\left[J+m(R+r)^{2}\right]\right\}^{1 / 2} \tau$ is the rescaled, dimensionless time, $\lambda=c_{p}\left\{\left(R \Omega^{2} / J r\right)\left[J+m(R+r)^{2}\right]\right\}^{1 / 2} /\left(2 m R r \Omega^{2}\right)$ is the dimensionless damping ratio, $k=$ $(R+r) T_{1} /\left[R \Omega^{2} \beta\left(J+m(R+r)^{2}\right)\right]$ is the dimensionless excitation amplitude, $\eta=$ $\omega\left\{\left(R \Omega^{2} / J r\right)\left[J+m(R+r)^{2}\right]\right\}^{-1 / 2}$ is the dimensionless excitation frequency, and (') denotes differentiation with respect to the rescaled time, $t$. Equation (9) is valid when the pendulum is not in contact with the constraints: i.e., for $|x|<1$. For impacts, i.e., for $|x|=1$, equation

(6) is also rescaled yielding

$$
\dot{x}^{+}=-e \dot{x}^{-} .
$$

Note that under the rescaling that led to equation (9) the rescaled anti-resonance frequency becomes $\eta_{A R}=\left\{1+(m / J)[R+r]^{2}\right\}^{-1 / 2}$. This is the point at which $\theta=0$ for the linear response: i.e., it is the desired operating point. 
The original equations of motion were in coupled form. Use of the aforementioned assumptions with the non-linear equations of motion resulted in a set of linear equations (7), (8) describing the motion of the pendulum between the constraints which were easily decoupled. In addition, the impact equations can be expressed in an uncoupled form (equation (6)), and consequently equations (9) and (10) fully describe the motion of the pendulum for a given set of initial conditions and parameter values. The dynamics of the carrier can then be determined by using equation (7) or (8). In particular, equation (7) yields

$$
\theta^{\prime \prime}=\left[m r(R+r) \phi^{\prime \prime}+T_{1} \sin \omega \tau\right] /\left[J+m(R+r)^{2}\right],
$$

and successive integrations with respect to time results in an expression for $\theta^{\prime}$, and $\theta$. In particular

$$
\theta=\left[m r(R+r) \phi-\left(T_{1} / \omega^{2}\right) \sin \omega \tau\right] /\left[J+m(R+r)^{2}\right]
$$

\section{IMPACTING PERIODIC RESPONSE}

The analysis for the existence and stability of periodic impacting motions can be carried out. It is noteworthy that the only type of impacting motions considered here is that of symmetric double-impact periodic motions (SDIP, for short) which have one impact for every half cycle of the excitation. To this effect, a Poincare section is defined with $\dot{x}=y$, as $\Sigma=\{(x, y, t) \mid x=+1, y>0\}$, along with a corresponding Poincaré map, $\mathbf{P}=\Sigma \rightarrow \Sigma$ or $\left(t_{i+1}, y_{i+1}\right)=\mathbf{P}\left(t_{i}, y_{i}\right)$, where the points $\left(t_{i}, y_{i}\right)$ are in $\Sigma$. These correspond to those states in which the pendulum is just coming into contact with the rigid constraint at $x=+1$. Periodic impacting motions in the phase space correspond to fixed or periodic points of the Poincaré map. If $(\bar{t}, \bar{y})$ is a fixed point corresponding to a SDIP motion of subharmonic order of $n$, then $(\bar{t}+2 \pi n / \eta, \bar{y})=\mathbf{P}(\bar{t}, \bar{y})$.

Necessary conditions for the existence of an SDIP motion can be obtained by matching conditions which require that a periodic, symmetric motion exists. The details are similar to those in references $[7,8]$ and are not given here. The conditions to be solved use the known linear solution for the motion of the pendulum during free flight and are given by

$$
\begin{array}{cl}
x(\bar{t}+\pi n / \eta ;+1, \bar{t},-e \bar{y})=-1, & \dot{x}(\bar{t}+\pi n / \eta ;+1, \bar{t},-e \bar{y})=-\bar{y}, \\
|x(t ;+1, \bar{t},-e \bar{y})|<1 & \text { for } t \in(\bar{t}, \bar{t}+\pi n / \eta),
\end{array}
$$

where $x\left(t ; x_{0}, t_{0}, y_{0}\right)$ is the explicit solution of equation (9) with initial conditions $\left(x_{0}, t_{0}, y_{o}\right)$. Equations $(11 \mathrm{a}, \mathrm{b})$ can be solved for $(\bar{t}, \bar{y})$. Each fixed point $(\bar{t}, \bar{y})$ of the Poincaré map which satisfies conditions (11) necessarily corresponds to a SDIP motion in the phase space. The analysis proceeds as in references [7-9] and results in a quadratic equation for $\bar{y}$, the velocity at impact at $x=+1$ for an SDIP motion. The resulting solutions for $\bar{y}$ are

$$
\bar{y}=J_{1}^{2} c^{2}\left[-\frac{(1+e) \bar{s}}{J_{1} c^{2} E} \pm \sqrt{\Delta}\right] /\left[\frac{\left(1+e^{2}\right) \bar{s}^{2}}{E^{2}}+\frac{J_{2}^{2}}{\eta^{2}}\right],
$$

and the corresponding phase at impact, $\bar{\phi}=\bar{t} \bmod (2 \pi / \eta)$, is given by

$$
c_{\eta}=\cos (\eta \bar{\phi}-\zeta)=\frac{(1+e) \bar{s}}{E c J_{1}} \bar{y}+\frac{1}{c}, \quad s_{\eta}=\sin (\eta \bar{\phi}-\zeta)=\frac{J_{2}}{c \eta J_{1}} \bar{y}
$$


where

$$
\begin{gathered}
\zeta=\tan ^{-1}\left(2 \lambda \eta /\left(1-\eta^{2}\right)\right), \quad \Lambda=\sqrt{1-\lambda^{2}} \\
\Delta=\left[E^{-2}(1+e)^{2} \bar{s}^{2}+\eta^{-2} J_{2}^{2}\left(1-c^{-2}\right)\right]\left(J_{1} c\right)^{-2}, \\
\bar{s}=\sin \Lambda \pi n / \eta \text { and } \bar{c}=\cos \Lambda \pi n / \eta, \quad E=\mathrm{e}^{-\lambda \pi n / \eta}, \\
\mathrm{J}_{1}=-\Lambda\left(1+\mathrm{E}^{-2}+2 \overline{\mathrm{c}} \mathrm{E}^{-1}\right), \\
\left.J_{2}=\Lambda E^{-1}(1-e) \bar{c}+\lambda E^{-1}(1+e) \bar{s}+\Lambda E^{-2}-\Lambda e, \quad c=k\left\{1-\eta^{2}\right)^{2}+4 \lambda^{2} \eta^{2}\right\}^{-1 / 2}
\end{gathered}
$$

$(13 \mathrm{~h}, \mathrm{i})$

These results for $(\bar{t}, \bar{y})$ are also valid for the simple one-degree-of-freedom impacting system in reference [8]. In reference [8] the free flight damping is taken to be zero. Thus, the present results extend those of reference [8] to more general, and more typical, cases.

The local stability of $(\bar{t}, \bar{y})$ can be studied by observing the eigenvalues of the $\overline{D P}$ matrix [8], i.e., the derivative of the Poincaré map evaluated at $(\bar{t}, \bar{y})$. Some details of the calculations and the explicit expressions for $\overline{D P}$ are provided in the Appendix. Any fixed point $(\bar{t}, \bar{y})$ resulting in eigenvalues of $\overline{D P}$ all with moduli less than unity is stable. Figure 3 is a typical plot of $\bar{y} v s$. $\eta$ depicting the stable and unstable response branches for $n=1$, while Figure 4 depicts a similar plot for the subharmonic orders $n=1,3$ and

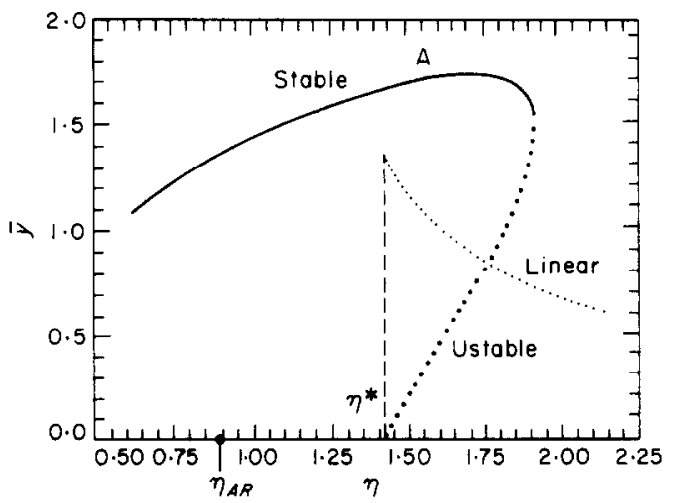

Figure 3. Linear and non-linear branches of the response of the pendulum. $k=1 \cdot 00, e=0 \cdot 10, \lambda=0 \cdot 00, n=1$, $\beta=22 \cdot 50$.

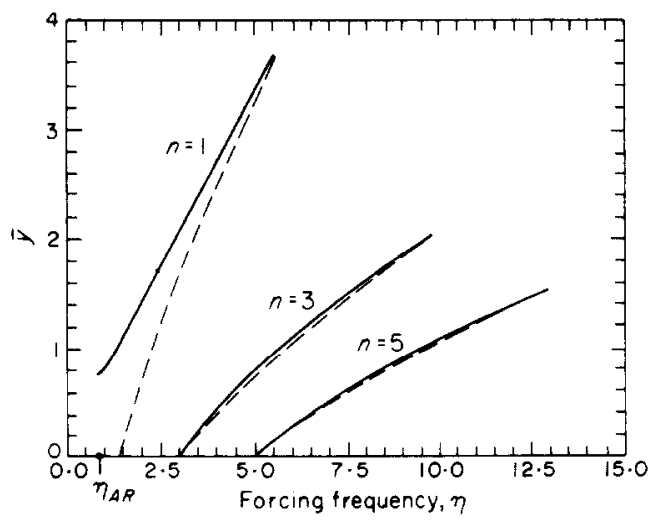

Figure 4. Primary $(n=1)$ and subharmonic $(n=3,5)$ responses of the pendulum.-, Stable; - - , unstable. $k=1 \cdot 00, e=0 \cdot 90, \lambda=0 \cdot 00$. 
5. (Note that, due to the symmetric nature of the SDIP motion, only odd orders of subharmonics can exist. The reason for this is that the excitation that takes the absorber from $x=+1$ to $x=-1$ should be equal and opposite of the one that brings the pendulum back to $x=+1$, implying $n=2 i+1$ ( $i$ integer) periods of the forcing are necessary to sustain a SDIP motion for one full cycle.) Superimposed on Figure 3 is the non-impacting, or linear, branch of the response curve. This branch corresponds to the maximum velocity (at $x=0$ ) of motions for which there are no impacts and the pendulum oscillates between the two constraints. Figures 5(a), 5(b), and 5(c) depict the variation of $\bar{y}$ as $k, \lambda$ and, $e$ are varied, respectively. Figure 6 illustrates the dynamic behavior of the pendulum and the carrier for a typical stable impacting motion, specifically point A in Figure 3.
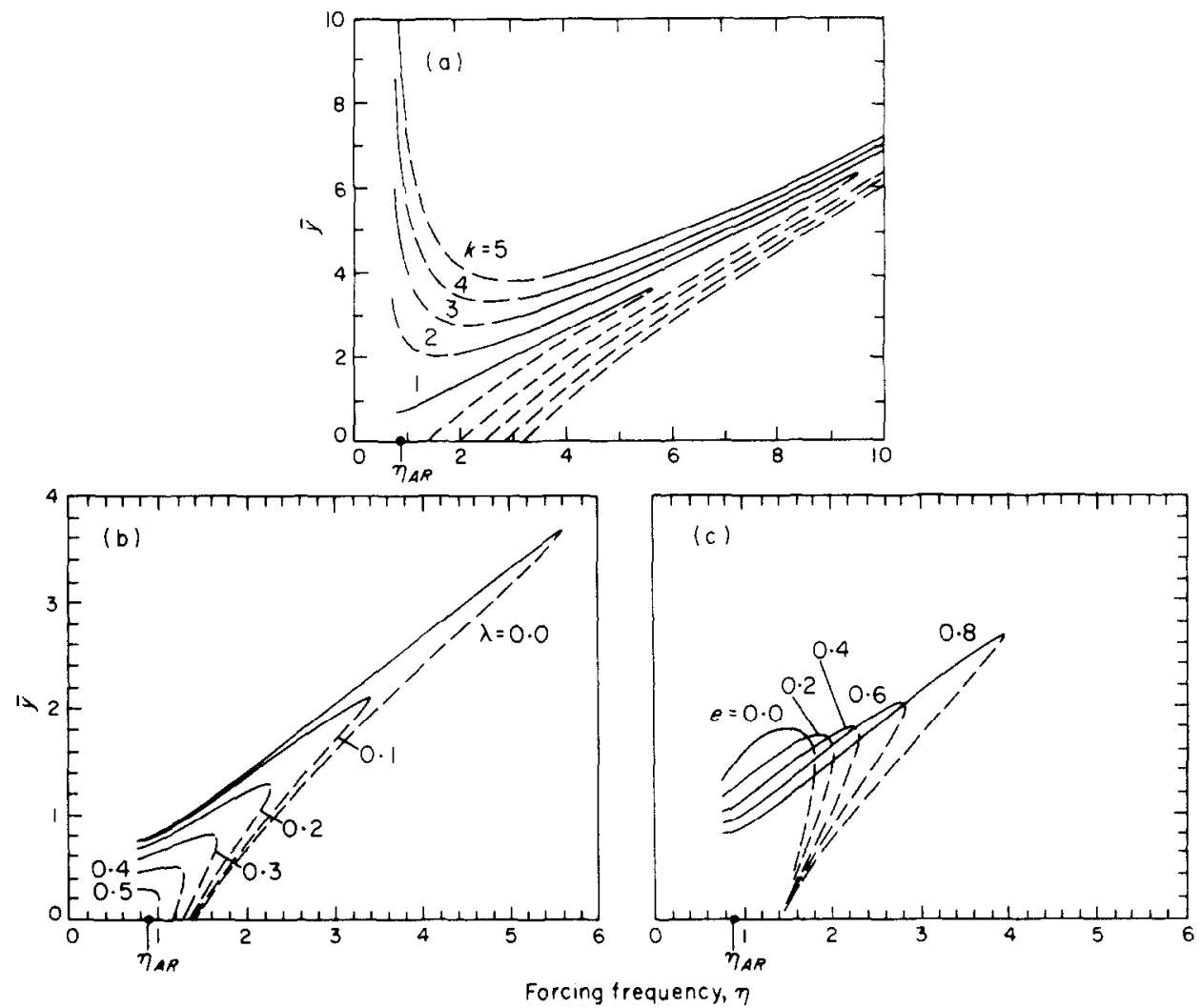

Figure 5. - Stable; - - , unstable. Effect of variation of (a) $k(e=0.90, \lambda=0.00,(\mathrm{~b}) \lambda(e=0 \cdot 90, k=1 \cdot 00)$ and (c) $e(k=1 \cdot 00, \lambda=0.00)$ on the pendulum response; $n=1 \cdot 00$.
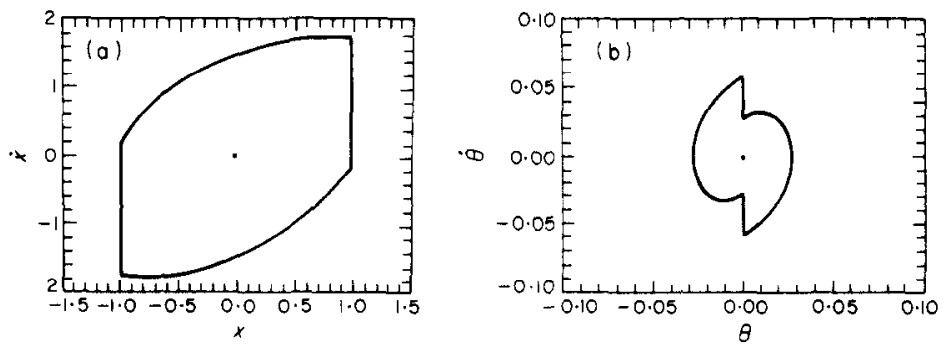

Figure 6. Phase trajectory of (a) the pendulum and (b) the carrier corresponding to point $A$ on Figure 3. $k=1 \cdot 00, e=0 \cdot 10, \lambda=0 \cdot 00, n=1 \cdot 00$. 
By referring to Figure 3 and moving horizontally from the right toward decreasing values of the frequency, $\eta$, one observes the following. For excitation frequencies around $\eta=2 \cdot 25$, the only possible motion is the linear one (or possibly the $n=3$ subharmonic) up to a point where stable and unstable SDIP with $n=1$ motions emerge in a saddle-node bifurcation [8]. This point corresponds to the excitation frequency for which $\Delta=0$, where $\Delta$ is as defined in equation (13d). Equivalently, in terms of the driving amplitude, these saddle-node bifurcations occur at

$$
k=J_{2} E\left\{\left[\left(1-\eta^{2}\right)^{2}+4 \lambda^{2} \eta^{2}\right] /\left[\left(1+e^{2}\right) \eta^{2} \bar{s}^{2}+J_{2}^{2} E^{2}\right]\right\}^{1 / 2} .
$$

As the frequency is decreased further, the steady-state amplitude, $y_{\text {max }}$, of the linear branch increases to a point where the motion just touches the constraints at $|x|=1$. This point is shown on the plot as $\eta^{*}$ at which the linear motion with $x_{\max }=1$ coexists along with an unstable SDIP motion having $\bar{y}=0$. That these two motions are in fact coincident at $\eta=\eta^{*}$ can be shown as follows. If the steady state amplitude of the linear branch is $1 \cdot 0$ then $c=1 \cdot 0$. For the minus branch of the SDIP motion with $n=1$, to satisfy $\bar{y}=0$ equation (12a) gives $\Delta^{1 / 2}+(1+e) \bar{s} / J_{1} c^{2} E=0$, or $-(1+e) \bar{s}=J_{1} c^{2} E \Delta^{1 / 2}$. It can be demonstrated that this condition holds for $c=1$ by using equations (13) as follows:

$$
J_{1} c^{2} E \Delta^{1 / 2}=\left\{c E\left[E^{-2}(1+e)^{2} \bar{s}^{2}+\eta^{-2} J_{2}^{2}\left(1-c^{-2}\right)\right]^{1 / 2}\right\}_{c=1}=-(1+e) \bar{s} .
$$

Additional decrease of the frequency results in the merging and annihilation of the (stable) linear and the (unstable) SDIP motions in a degenerate saddle-node bifurcation at $\eta=\eta^{*}$.

A point of interest about the transformation of linear motions into impacting SDIP orbits is that, as the amplitude of the oscillation of the absorber is increased in the linear (non-impacting) range, eventually the amplitude, $|x|$, will equal 1.0 ; i.e., the motion just grazes the stops while still retaining its linear character. A further attempt to increase the amplitude of the linear motion of the absorber by decreasing $\eta$ will destroy the nonimpacting motion. However, contrary to what one might intuitively expect, such transformation from linear to non-linear orbits will not be a smooth one and there will be a jump discontinuity. In other words, a stable SDIP motion with $\bar{y} \simeq 0$ will not emerge from the linear motion as $\eta$ is decreased past $\eta^{*}$. Rather another impacting motion which is not a simple continuation of the linear motion for $\eta<\eta^{*}$ will appear instead. This occurs since the stable linear motion with $x_{\max }=1, y=0$ and an unstable SDIP motion with $\bar{y}=0$ annihilate each other in a saddle-node bifurcation. The overall result is that as parameters are varied, a linear motion does not smoothly transform into an SDIP motion.

It is possible for the stable SDIP response to become unstable in a symmetry-breaking pitchfork bifurcation which results in an antisymmetric pair of periodic motions. These motions can, as $\eta$ is decreased further, undergo period doubling bifurcations which result in chaotic dynamics for the system. This is described in more detail in section 4 .

It is worth mentioning that, for very low frequencies of the excitation $\eta$, the results corresponding to a stable SDIP motion are in fact what are called "penetrating" motions which are consistent with the mathematical modeling of the system but are physically not realizable [8]; they satisfy conditions (11a) and (11b) but violate condition (11c).

An extensive study was carried out to determine the possibility of coexistence of impacting and non-impacting motions at the anti-resonance point. To this effect, a fine grid of points in the parameter space $(k, \lambda, e)$ was searched to detect the occurrence of such coexistence. Figure 7 is a plot showing the coexistence points for different values of $k$ in the $(\lambda, e)$ space.

The occurrence of coexistence at the anti-resonance point is crucial from a design point of view since the dynamic behavior of the pendulum, and consequently of the carrier, 


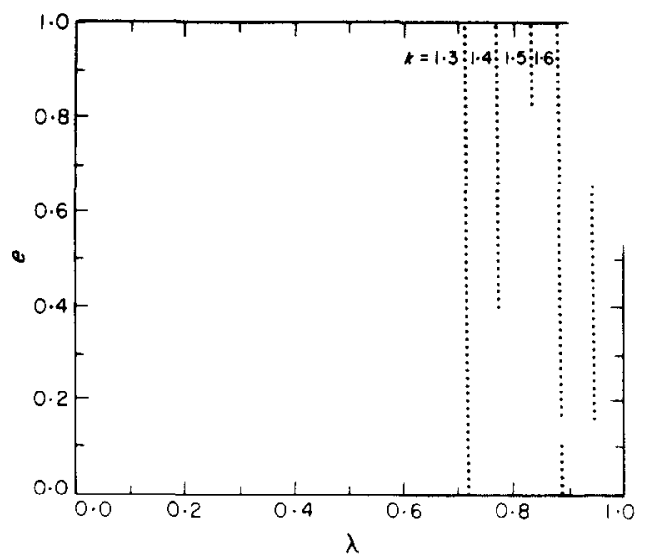

Figure 7. Regions of coexistence at the anti-resonance frequency in the parameter space $(k, \lambda, e)$.

depend on the initial conditions and slight disturbances could take the motion from non-impacting to impacting and vice versa. Coexistence at the anti-resonance point occurs only at unreasonably large values of the pendulum damping ratio, $\lambda>0 \cdot 7$. This places the issue outside the realm of practical applications. In addition, coexistence is found only for $k$ values above 1.0 which is well above the $k$ range for which the desired linear motion exists. This is easily seen by requiring $c<1$ at $\eta=\eta_{A R}$ which results in the requirement $k<k_{c r}(\lambda)=\left\{\left(1-\eta_{A R}^{2}\right)^{2}+4 \lambda^{2} \eta_{A R}^{2}\right\}^{1 / 2}$ for existence of the linear motion. The function $k_{c r}(\lambda)$ is bounded above by $0 \cdot 50$ for $0 \leqslant \lambda \leqslant 0 \cdot 25$.

Figure 10 depicts a case revealing the coexistence of all three types of motions, namely, stable and unstable SDIP and linear non-impacting motions at $\eta=\eta_{A R}$.

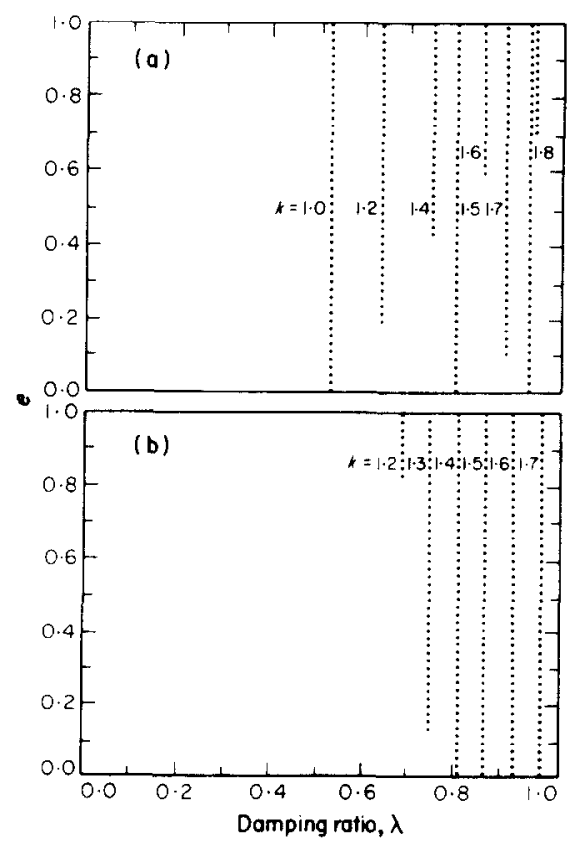

Figure 8 . Regions of coexistence at (a) $+5 \%$ detuning, (b) $-5 \%$ detuning. 
The effect of "detuning" can also be studied in this context. Detuning refers to the presence of disturbances with a slightly different excitation frequency than the frequency for which the absorber is designed. The primary focus of the study of detuning in this report is its effect on the probability of the occurrence of coexistence at the operating frequency. In other words, if the excitation frequency is not exactly at the anti-resonance point, $\eta_{A R}$, will this increase the likelihood of coexistence? Figures 8 and 9 , which are similar to Figure 7 , were obtained in the following manner. The operating excitation frequency in Figure 8 has a $\pm 5 \%$ variation from the anti-resonance frequency and that of Figure 9 has a $\pm 10 \%$ variation. As can be observed from these figures, the effect of detuning has a slight tendency to increase the possibility of coexistence. However, it still does not lead to coexistence at reasonable values of the damping ratio $\lambda$. This lack of sensitivity implies that tuned CPVA systems should have no problems with steady state impacting motions.

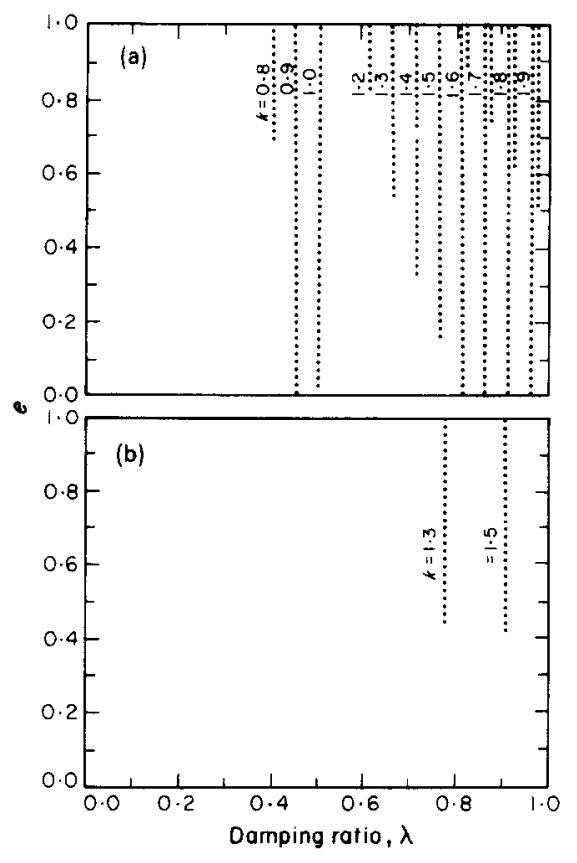

Figure 9. Regions of coexistence at (a) $+10 \%$ detuning, (b) $-10 \%$ detuning.

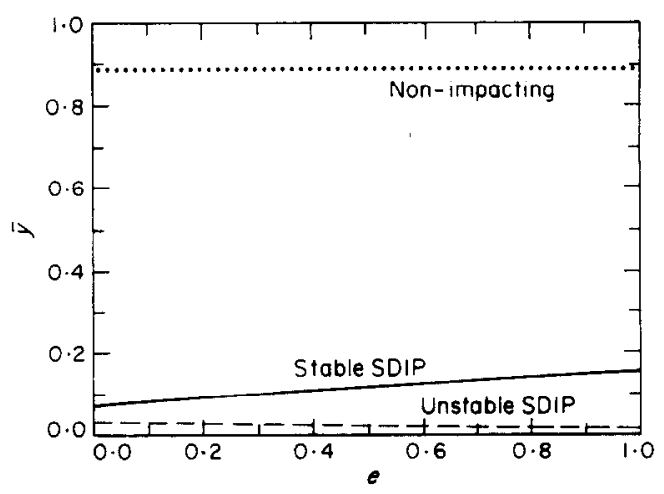

Figure 10. Types of coexistence at the anti-resonance frequency. $k=1 \cdot 30, \lambda=0 \cdot 72, n=1 \cdot 00$. 


\section{NON-LINEAR RESPONSES AND CHAOTIC MOTIONS}

As mentioned earlier, in addition to the degenerate saddle-node bifurcations that result in the "jump phenomena" (see Figures 3-5), another form of bifurcation can occur which results in a change of stability of the SDIP response. For instance, for $k=2 \cdot 0, e=0.95$ and $\lambda=0.01$, the frequency response is as shown in Figure 11 and it is observed that as the excitation frequency is decreased from $\eta=2.0$ the upper SDIP branch becomes unstable at about $\eta=1.60$; this is indicated as point $B$ in Figure 11. For excitation frequencies between $\eta \approx 1.60$ and $\eta \approx 1.0$, where the latter frequency is close to the anti-resonance frequency $\left(\eta_{A R}=0.89\right)$, there exist no stable SDIP or linear non-impacting periodic motions. However, numerous simulations along with detailed analysis based on center manifold theory [8] reveal that as the excitation frequency is decreased past point $\mathrm{B}$, an eigenvalue of the $\overline{D P}$ matrix passes out of the unit circle through +1 which renders the response unstable. At this frequency a super-critical pitchfork bifurcation takes place in which the stable SDIP motion becomes unstable and a pair of stable anti-symmetric double impact periodic orbits are generated. These motions then each undergo a succession of period doubling, or flip, bifurcations, which eventually result in non-periodic, or chaotic motions.

Figure 12 depicts the phase trjactory of an unstable SDIP motion along with a pair of stable anti-symmetric period-one double impact motions and Figure 13 illustrates their corresponding time responses. Plots showing the succession of period-doublings of similar

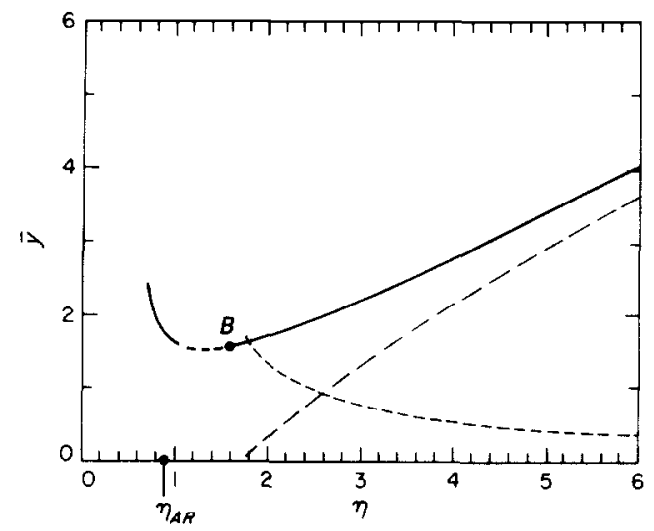

Figure 11. Frequency response of the pendulum. $k=2 \cdot 00, e=0 \cdot 95, \lambda=0 \cdot 01, n=1 \cdot 00$.

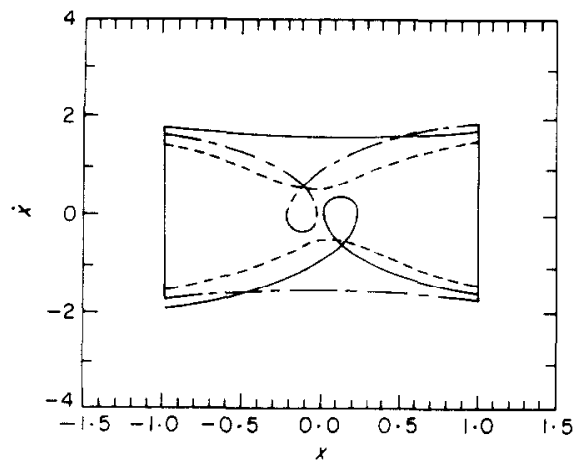

Figure 12. Phase trajectory of an unstable SDIP coexisting with a pair of anti-symmetric double-impact periodic motions. $k=2.00, e=0.9500, \lambda=0.0100, n=1 \cdot 000, \eta=1.4010$. 

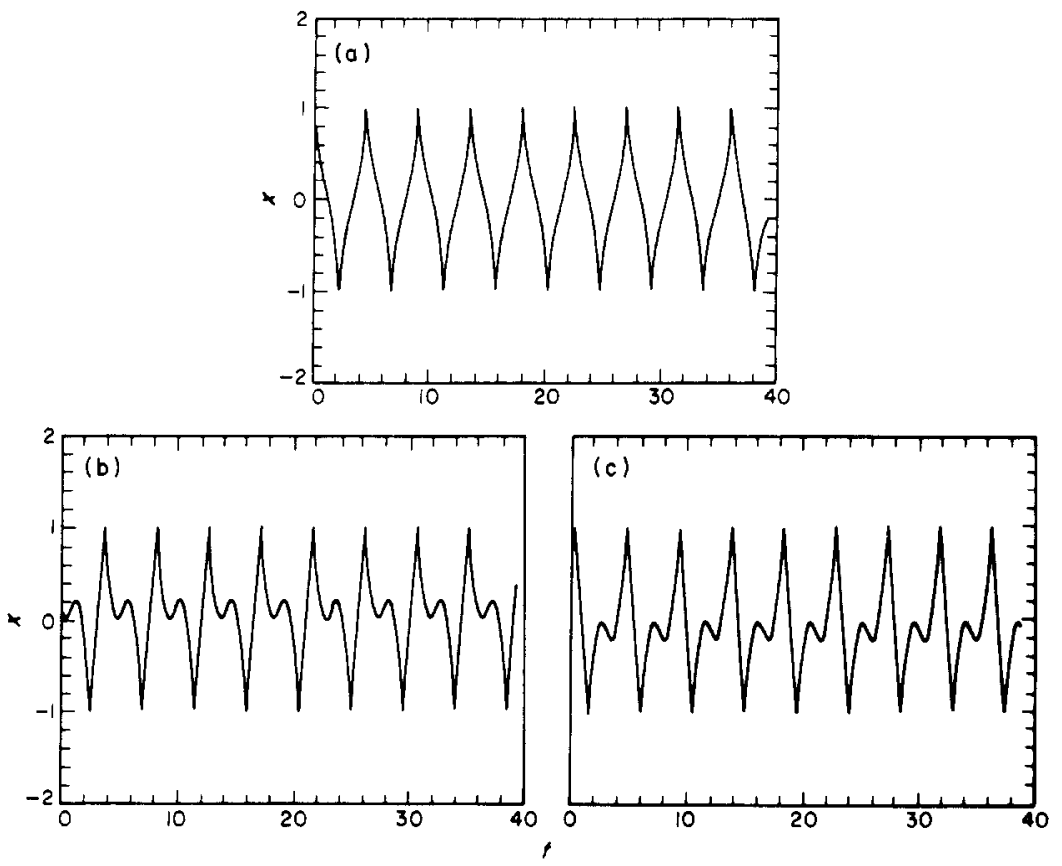

Figure 13. (a) Time response of an unstable SDIP; (b) time response of a stable anti-symmetric double-impact periodic orbit; (c) time response of a stable anti-symmetric double-impact periodic orbit. Parameters as Figure 12.

anti-symmetric motions can be found in reference [8]. Figure 14(a) is a Poincaré map indicating the existence of a strange attractor which corresponds to a chaotic motion; this occurs after the completion of the period doubling sequence [10]. Figure 14(b) depicts a portion of a typical time response of the absorber mass for an initial condition within the strange attractor.

While chaotic motions may exist at frequencies close to the anti-resonance frequency, no chaos was found at anti-resonance for absorbers designed to operate within their linear range. However, certain disturbances may lead to chaotic responses. In particular, inputs can lead to chaotic motions near a response curve of subharmonic order $n$ in ranges from $\eta=n$ to a point analogous to $\mathrm{B}$ in Figure 11. The system is non-linear and
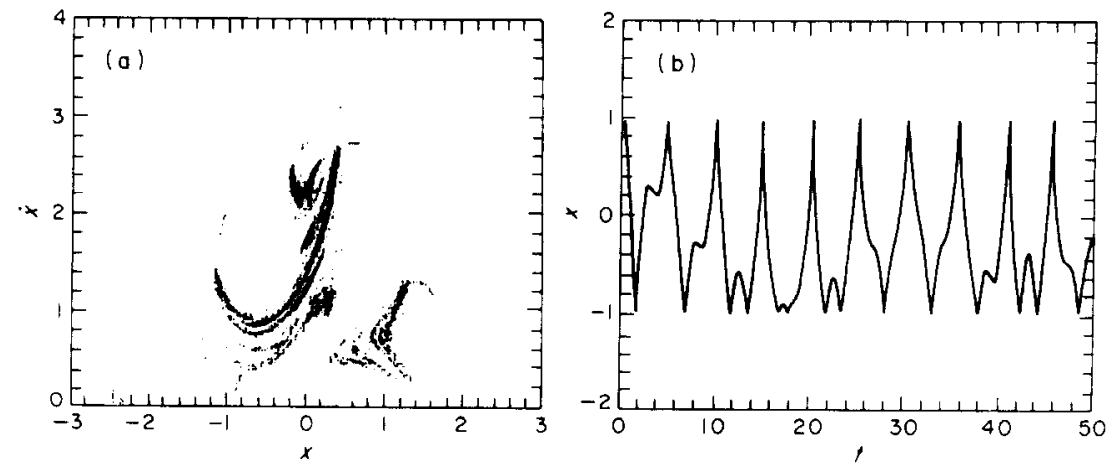

Figure 14. (a) Poincaré map indicating chaos; (b) time response of the pendulum with initial condition inside the strange attractor set. Parameters as Figure 12 except $\eta=1 \cdot 2200$. 
one cannot rule out these types of effects from disturbances, even if they are small, since superposition does not apply when impacts occur.

\section{DISCUSSION}

The main conclusion of this work is that motion-limiting stops can be effectively employed when placed at amplitudes larger than the steady state response predicted from the linear system at the anti-resonance frequency. This is so due to the fact that steady state impacting motions can occur at the anti-resonance frequency only if the damping between the pendulum and carrier is unreasonably large. The impact dynamics of the system can be very complicated when it is subjected to frequencies above anti-resonance; this can include chaotic motions and/or a variety of periodic responses.

A limiting factor in many designs of the CPVA is that the steady state pendulum angle amplitudes must become large when the disturbing torque amplitudes are large. If the system is driven out of the region of validity for linearization, then non-linear effects can lead to catastrophic failures $[5,12]$. This is currently dealt with by making the effective pendulum path non-circular. Cycloidal paths are common in helicopter applications; more optimal paths are currently being worked on in the automotive industry [6].

If stops are not employed at all, and the pendulum is allowed to swing over the top (an extreme situation which is rarely, if ever, seen in applications), the system can undergo chaotic motions in which the pendulum undergoes chaotic sequences of clockwise and counter-clockwise rotations [11].

Recent experiments have been carried out on a simple, non-rotating, impacting pendulum which is governed by the same equations of motion as the present system (equations (9), (10)). These demonstrated the existence of SDIP motions, pitchfork bifurcations and chaotic dynamics [13]. Frequencies near the corresponding anti-resonance frequency were not, however, attainable in that system since $\eta$ was restricted to be unity or higher by equipment limitations.

The SDIP motions are a very specific type of periodic motion. An infinite number of other types of periodic motions exist for this system (this is known since the presence of chaotic dynamics indicates the existence of horeshoe sets for the map $P$ which in turn contain this infinity of periodic motions along with non-periodic motions [10]). Thus a complete study of the impacting dynamics is out of the question. However, SDIP motions are the most common; this has been observed in experiments [13] and simulations. In addition, they are a good indicator of where other impacting motions exist since many (although not all) of the other types arise out of bifurcations which are directly tied to an SDIP motion.

\section{REFERENCES}

1. C. F. TAYLOR 1982 The Internal Combustion Engine in Theory and Practice, Vol. 2: Combustion, Fuels, Materials, Design. Cambridge, Massachusetts: M.I.T. Press.

2. J. P. Den Hartog 1938 in S. Timoshenko 60th Anniversay Volume, 17-26. Tuned pendulums as torsional vibration absorbers. New York: MacMillan Co.

3. W. T. THOMSON 1981 Theory of Vibration with Applications (2nd edition). Englewood Cliffs, New Jersey: Prentice-Hall.

4. F. R. E. CROSSLEY 1985 Journal of Applied Mechanics 75, 41-47. The forced oscillation of the centrifugal pendulum with wide angles.

5. D. E. NEWLAND 1963 Ph.D. Thesis, Massachusetts Institute of Technology. Nonlinear vibrations: a comparative study with applications to centrifugal pendulum vibration absorbers.

6. FORD MOTOR CO. Reports on Centrifugal Pendulum Vibration Absorber (unpublished). 
7. S. F. MASRI 1972 Journal of Applied Mechanics 39, 563-568. Theory of dynamic vibration neutralizer with motion limiting stops.

8. S. W. SHAW 1985 Journal of Applied Mechanics 52, 453-458. The dynamics of a harmonically excited system having rigid constraints, part $\mathrm{I}$ : subharmonic motions and local bifurcations.

9. S. W. SHAw 1986 Journal of Sound and Vibration 108, 305-325. On the dynamic response of a system with dry friction.

10. S. W. SHAW 1985 Journal of Applied Mechanics 52, 459-464. The dynamics of a harmonically excited system having rigid constraints, part II: chaotic motions and global bifurcations.

11. S. W. SHAW and S. WIGGINS 1988 American Society of Mechanial Engineers Journal of Applied mechanics to appear). Chaotic motions of a torsional vibration absorber.

12. D. E. NEWLAND 1964 American Society of Mechanical Engineers Journal of Engineering for Industry 86, 257-263. Nonlinear aspects of the performance of centrifugal pendulum vibration absorbers.

13. D. W. MOORE and S. W. SHAW 1988 Michigan State University Preprint. The dynamic response of an impacting pendulum.

\section{APPENDIX}

\section{A1. DIMENSIONS OF THE CPVA MODEL.}

The dimensions of the CPVA model used for numerical calculations in this report are the same as those used by Newland [12]: pendulum mass, $m=25.261 \mathrm{~b} \mathrm{~m}$; pendulum length, $r=0.561 \mathrm{in}$; carrier radius, $R=2.24 \mathrm{in}$; carrier polar moment of inertia, $J=$ $772.28 \mathrm{lbm}$ in in; carrier steady velocity, $\Omega=1000 \mathrm{rpm}$; constraint angle (from one constraint to the other): $2 \beta=45^{\circ}$.

A2. CALCULATIONS FOR THE STABILITY MATRIX, $\underline{\overline{D P}}$

The stability of periodic points can be investigated by tracing in time the dynamics of perturbations on the initial conditions from the periodic point $(\bar{t}, \bar{y})$. This procedure can be discretized by observing the effects of perturbations by using the Poincaré map. In other words, a slight disturbance $(t, y)=(\bar{t}+\zeta, \bar{y}+\nu)$ is imposed on the periodic point and its state is then observed after subsequent returns to the Poincare section. Since local stability is of interest here, linearizing one obtains $\left(\zeta_{i+1}, \nu_{i+1}\right)=\overline{D P}\left(\zeta_{i}, \nu_{i}\right)$, where $(\zeta, \nu)$ is the perturbation and $\overline{D P}$ is the first derivative of the Poincaré map, $P$, evaluated at the periodic point $(\bar{t}, \bar{y})$. By using the notations employed in Figure A1 the $\underline{\overline{D P}}$ matrix can be written as

$$
\underline{\overline{D P}}=\underline{D P}(\bar{t}, \bar{y})=\left[\begin{array}{ll}
\partial t_{E} / \partial t_{A} & \partial t_{E} / \partial y_{A} \\
\partial y_{E} / \partial t_{A} & \partial y_{E} / \partial y_{A}
\end{array}\right]_{(\bar{i}, \bar{y})} \triangleq\left[\frac{\partial\left(t_{E}, y_{E}\right)}{\partial\left(t_{A}, y_{A}\right)}\right]_{(\bar{i}, \bar{y})} .
$$

Hence, the problem of stability of periodic points is reduced to the study of the eigenvalues of the $\underline{\overline{D P}}$ matrix. Since explicit expressions for the Poincare map are not available, the

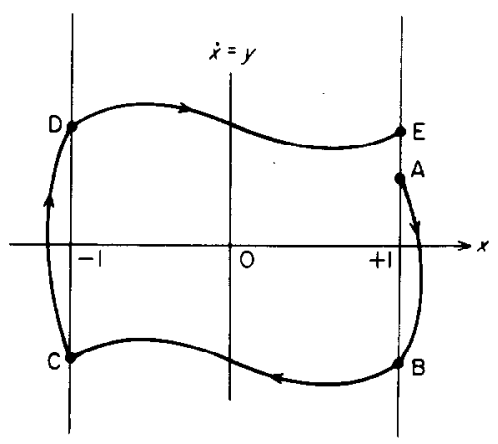

Figure A1. A typical phase trajectory of the pendulum with double impacts. 
following implicit differentiation procedure is used to obtain an expression for the matrix $\underline{\overline{D P}}$; see references $[8,9]$ for similar calculations.

For SDIP motions, the time and velocity at point B (see Figure A1) uniquely determine those of point $C\left(t_{C}, y_{C}<0\right)$ by

$$
x\left(t_{C} ;+1, t_{B}, y_{B}\right)=-1, \quad \dot{x}\left(t_{C} ;+1, t_{B}, y_{B}\right)=y_{C}
$$

where $x\left(t_{C} ;+1, t_{B}, y_{B}\right)$ is the explicitly known solution of equation (9) based at point $B$. Equation (A2a) can be, in principle, inverted to yield $t_{C}=t_{C}\left(t_{B}, y_{B}\right)$, and substituting into equation (A2b) gives $y_{C}=y_{C}\left(t_{B}, y_{B}\right)$.

Continuing the motion from $C$ to $D$ one can write $y_{D}=-e y_{C}, t_{D}=t_{C}$, and the motion is again governed by equation (9) from point $D$ to $E$, with the time and velocity at $E$ being uniquely determined from those at $D$. The corresponding conditions in this case are $x\left(t_{E} ;-1, t_{D}, y_{D}\right)=+1, \dot{x}\left(t_{E} ;-1, t_{D}, y_{D}\right)=y_{E}$, which, when inverted, yield $t_{E}=$ $t_{E}\left(t_{D}, y_{D}\right), y_{E}=y_{E}\left(t_{D}, y_{D}\right)$.

From the above analysis one can observe that, the time and velocity at point $A$ can uniquely determine the time and velocity at $E$ : i.e., $t_{E}=t_{E}\left(t_{A}, y_{A}\right), y_{E}=y_{E}\left(t_{A}, y_{A}\right)$. Hence, using the chain rule and the notations defined in equation (A1) one can write

$$
\overline{\overline{D P}}=\left[\frac{\partial\left(t_{E}, y_{E}\right)}{\partial\left(t_{A}, y_{A}\right)}\right]=\left[\frac{\partial\left(t_{E}, y_{E}\right)}{\partial\left(t_{D}, y_{D}\right)}\right]\left[\frac{\partial\left(t_{D}, y_{D}\right)}{\partial\left(t_{C}, y_{C}\right)}\right]\left[\frac{\partial\left(t_{C}, y_{C}\right)}{\partial\left(t_{B}, y_{B}\right)}\right]\left[\frac{\partial\left(t_{B}, y_{B}\right)}{\partial\left(t_{A}, y_{A}\right)}\right] \text {. }
$$

It is easily seen that

$$
\left[\frac{\partial\left(t_{B}, y_{B}\right)}{\partial\left(t_{A}, y_{A}\right)}\right]=\left[\frac{\partial\left(t_{D}, y_{D}\right)}{\partial\left(t_{C}, y_{C}\right)}\right]=\left[\begin{array}{cc}
1 & 0 \\
0 & -e
\end{array}\right] \text {. }
$$

The remaining two matrices can be computed by using implicit differentiation.

The final expression for the $\underline{\overline{D P}}$ matrix is

$$
\begin{gathered}
\underline{\overline{D P}}=\left[d_{i j}\right], \quad i, j=1,2, \\
d_{11}=k_{1} k_{2}-e k_{3} k_{4}, \quad d_{12}=-e k_{1} k_{5}+e^{2} k_{3} k_{6}, \\
d_{21}=k_{2} k_{7}-e k_{4} k_{8}, \quad d_{22}=-e k_{5} k_{7}+e^{2} k_{6} k_{8}, \\
k_{1}=(E / \Lambda \bar{y})\left[\bar{s}-c c_{\eta} \bar{s}+2 c \lambda \eta s_{\eta} \bar{s}+e \bar{y} \Lambda \bar{c}+c \eta^{2} c_{\eta} \bar{s}-e \bar{y} \lambda \bar{s}\right], \\
k_{4}=(1-2 \lambda \bar{y}-K \cos \eta \bar{t}) k_{2}+(E / \Lambda)\left(-\gamma \bar{s}+c \lambda c_{\eta} \bar{s}-\left(1-2 \lambda^{2}\right) e \bar{y} \bar{s}\right. \\
k_{2}=-(E / \Lambda \bar{y})\left[-\bar{s}+c c_{\eta} \bar{s}+\lambda e \bar{y} \bar{s}-2 c \lambda s_{\eta} \bar{s}-e \bar{y} \Lambda \bar{c}-c \eta^{2} c_{\eta} \bar{s}\right], \quad k_{3}=-(E / \Lambda \bar{y}) \bar{s} \\
k_{7}=(k \cos \eta \bar{t}-2 \lambda \bar{y}-1) k_{1}+(E / \Lambda)\left(\lambda \bar{c}-c \lambda c_{\eta} \bar{s}+e \bar{y}\left(1-2 \lambda^{2}\right) \bar{s}\right. \\
+2 e \bar{y} \Lambda \lambda \bar{c}-\left(1-2 \lambda^{2}\right) c \eta s_{\eta} \bar{s}-2 c \Lambda \lambda \eta s_{\eta} \bar{s}+2 c \Lambda \lambda \eta s_{\eta} \bar{c}+\Lambda \bar{c}-c \eta s_{\eta} \bar{s}-c \Lambda c_{\eta} \bar{c}-c \gamma \eta^{2} c_{\eta} \bar{s}+c \Lambda \eta^{2} c_{\eta} \bar{c}, \\
\left.k_{5}=(E \bar{s}) / \Lambda \bar{y}\right)-k_{3}, \quad k_{6}=\left(1+2 \lambda \bar{y}-k \Lambda c_{\eta} \bar{c}+c \lambda \eta^{2} c_{\eta} \bar{s}-c \Lambda \eta^{2} c_{\eta} \bar{c}\right)
\end{gathered}
$$

Expressions for $\bar{s}, \bar{c}, \bar{y}, s_{\eta}, c_{\eta}$, etc., can be found in section 3 of the paper. 